\title{
Integrated Photodiodes in Nanometer CMOS TECHNOLOGIES
}

\author{
Mohamed Atef \\ Senior Member IEEE \\ Electrical Engineering Department, Assiut University, Assiut, Egypt, \\ moh_atef@au.edu.eg
}

\begin{abstract}
The main speed limitations of standard nanometer CMOS photodiodes are coming from the substrate slow carriers diffusion. Also the capacitance of the photodiode is increasing with technology scaling as the doping is increasing. The PD capacitance must be as low as possible to reduce the high frequency loss of the photocurrent to reach highest possible sensitivity. These demands are partially conflicting; so a trade-off is necessary, especially due to the wavelength dependence of the penetration depth. Many photodiode structures are recently introduced to increase the photodiode intrinsic speed in nanometer CMOS technology. In this paper a theoretical and comparative study for different recently published photodiodes structures fabricated in nanometre CMOS technology will be presented. This paper gives the researchers a detailed comparison and analysis to select the right photodiode to achieve the best performance in challenging nanometre CMOS technology.
\end{abstract}

Keywords: Integrated Photodiode,, DPD, SML, Interdigitated, PIN, APD, Nanometer CMOS Technology.

\section{Introduction}

Process modifications are needed to optimize the performance of both PIN-PD and BJT in BiCMOS technology. The integrated photodiode needs a thick lowly doped intrinsic layer whereas the BJT needs a thin higher doped layer for the $\mathrm{n}$ collector. BiCMOS PIN-PDs achieve higher performance than the CMOS PDs at the expense of a more complex and expensive technology [1, 2]. Silicon on Insulator (SOI) technology builds the transistors on a very thin layer of silicon. An insulating layer is placed between the thin layer of silicon and the substrate. The buried oxide layer prevents the slowly diffusing carriers generated in the substrate to reach the photodiode implemented on the thin silicon layer, see Fig.1. Photodetectors implemented in SOI have a higher speed compared to standard CMOS photodetectors. On the other hand, a low responsivity is expected, especially at long wavelengths, due to the large percentage of substrate generated carriers which will not contribute to the photodiode current. Also SOI technology involves higher costs than their standard CMOS counterparts.

The low cost is the main motivation for choosing silicon CMOS technology for integrated photodiodes. The new nanometer CMOS technologies are expensive. But in a few years, the high manufacturing cost will be reduced by the large production volumes. The trend towards optical interconnects for rack-to-rack and chip-to-chip connects instead of wire communications will increase the mass production volumes and reduce the production costs.

Standard CMOS technology is the cheapest silicon technology because of the lower number of production masks and large volume production compared to BiCMOS and SOI technologies. The main challenge of choosing standard nanometer CMOS for light detection is that the technology is 
not optimized for optoelectronic devices. Moreover, as the technology scaled down the layers' doping levels were increased and the supply voltage is decreased. The technology down scaling will increase the disadvantages of the integration of photodiodes in newer CMOS technologies because of four reasons. First, the width of the space-charge region is small due to the high doping and the limited bias voltage which does not match the penetration depth of light in silicon. This will lead to slow photodiodes due to the large diffusion components in the photocurrent, ass illustrated in Fig.1. As the maximum junction width decreases by technology down scaling the dynamic responsivity will be reduced because the internal dynamic quantum efficiency will decrease [1]. Second, the limited biasing voltage and the high doping have the effect to increase the junctions' capacitance and as a result the bandwidth will be decreased.

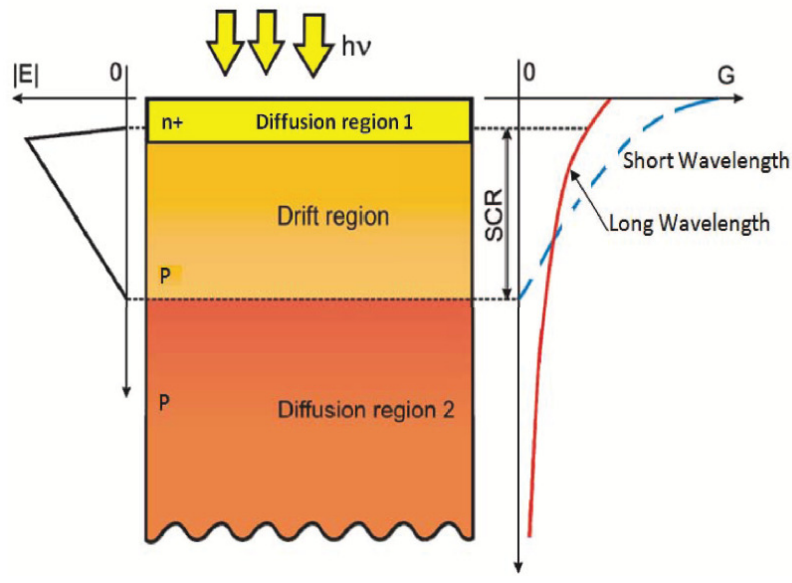

Fig 1: Drift and diffusion regions with electric field and photo-generation distribution in photodiode

Third, as the technology scaled down the number of metal and insulation layers increased which increased the optical reflection and optical interference within the insulator stack; this decreased the optical quantum efficiency and so the responsivity as well as increased the spectral fluctuation in it. Finally, the design rules for metal density per area become more restricted as the technology scaled down. As a result unwanted metal layers must be distributed over the photodiode to fulfill the metal density rules. This reduces the photodiode's active area and part of the light will be reflected from the undesired metal regions and the responsivity will decrease. The above limitations result in integrated photodiodes in nanometer CMOS with smaller responsivity and lower speed compared to commercially available discrete diodes or photodiodes integrated in other technologies as e. g. OPTO BiCMOS technologies. We should overcome these CMOS photodiode limitations to implement cheap but high performance integrated optical receivers.

In the coming section different photodiode structures will be introduced to enhance the speed and responsivity problems in nanometer CMOS technologies. The fingered PD can be used to increase the electric field near the surface and so the diffusion current will be decreased. Spatially modulated light (SML) detectors can be used to eliminate the substrate diffusion current. Both fingered and SML PDs increase the PD speed and also solve the problem of the metal density; the metals are distributed over all the PD area. Avalanche PDs (APDs) can be implemented to achieve a high responsivity and an extended bandwidth due to the involved inductive behavior due to the avalanche effect. An anti-reflection coating (ARC) can be used to reduce the light reflection from the insulator stack and it can be optimized for a certain wavelength. 


\section{Photodiode Speed}

The main speed limitations of standard nanometer CMOS photodiodes are coming from the substrate slow carriers diffusion generated in region 2 (see Fig.1). Also the capacitance of the photodiode must be as low as possible to keep the high frequency loss of the photocurrent low to reach highest possible sensitivity. These demands are partially conflicting; so a trade-off is necessary, especially due to the wavelength dependence of the penetration depth. Many photodiode structures are introduced to increase the photodiode intrinsic speed in nanometer CMOS technology.

To get the highest speed, the thickness of the SCR of the photodiode must be maximized. For a good trade-off, the main parameters that must be known are the maximum data rate, the operating wavelength, type of photodiode and the maximum voltage available for the photodiode. A main characteristic of integrated optical receivers is the achievable sensitivity, which depends strongly on the responsivity of the photodiode and also the capacitance, so minimum capacitance and maximum responsivity lead to maximum sensitivity. This could be achieved by maximizing the thickness of the SCR by increasing the reveres biasing voltage. But this contradicts to speed, since increasing the SCR

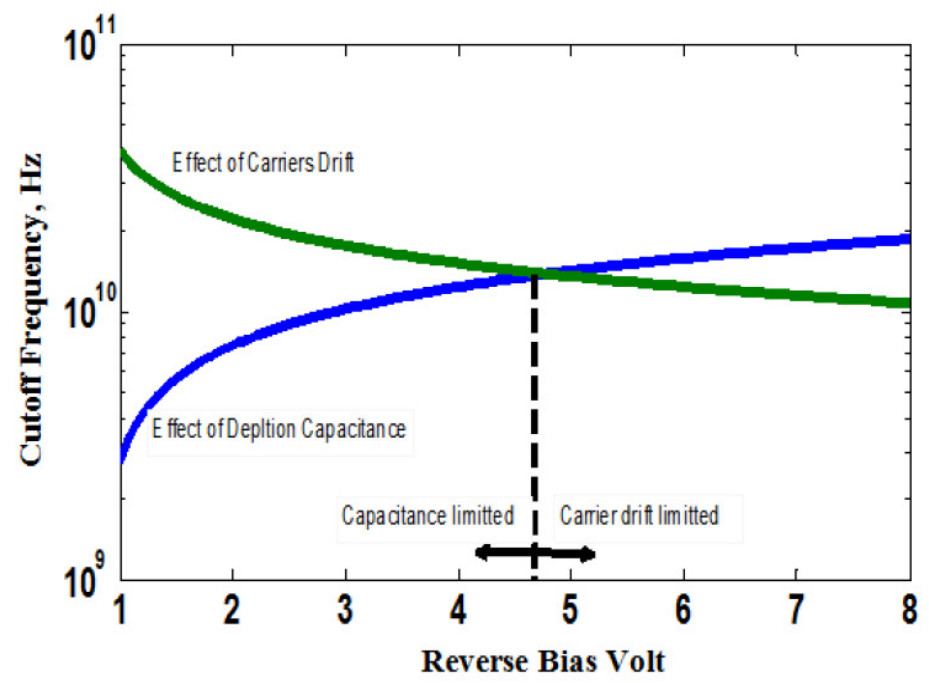

Fig. 2: Electron drift cutoff frequency as a function of the reverse biasing volt.

layer thickness increases the distance the generated carriers have to drift and so the drift transition time of the carriers increases, see Figure 2. As in nanometer CMOS technology the substrate doping level and the wells are in the range of $10^{15}$ and $10^{18}$ respectively, the SCR width will be small and the diffusion current will dominant the drift current(see Fig.1). So, the drift time will be negligible to the diffusion time. As a result the maximization of SCR is the optimum way to get the maximum speed and responsivity

The diffusion distance of electron in P-substrate dp can be defined as the distance at which $90 \%$ of the incident optical power is absorbed $\left(\mathrm{d}_{\mathrm{p}}=2.3 / \alpha\right)$. The time tdiff, $\mathrm{n}$ for the diffusion of electrons in the P-substrate anode (region 2 in Fig 1) with dp diffusion distance toward SCR can be calculated by: 


$$
t_{d i f f, n}=\frac{d_{p}^{2}}{2 D_{n}}=\frac{d_{p}^{2} q}{2 \mu_{n} K T}=\frac{\left(\frac{2.3}{\alpha}\right)^{2} q}{2 \mu_{n} K T}
$$

The intrinsic bandwidth of the photodiode due to the diffusion of electron in P-substrate can be estimated by:

$$
B W_{c, \text { in }} \approx \frac{2.4}{\pi\left(t_{d i f f, n}\right)}=\frac{2.4}{\pi\left(t_{d i f f, n}\right)}
$$

Figure 3 shows the electron diffusion bandwidth as function of doping for different wavelengths. The longer the wavelength the deeper the photocarriers are generated and a more time is needed to diffuse from the neural region toward the SCR. Generally spoken for short optical wavelengths (e.g. $1=410 \mathrm{~nm}$ ) surface effects are dominant. For short wavelengths diffusion in the surface electrode (region 1) can dominate, which should then be thin to reduce carrier diffusion. For long optical wavelengths (e.g. $1=850 \mathrm{~nm}$ ) charge carriers are generated deep in the substrate (region 2), where no electric field is present. Diffusion should be avoided where possible, since the effect causes the photodiode to slow down. Often a photodiode must be optimized for a particular wavelength to suppress carrier diffusion to get a higher intrinsic bandwidth. To decrease the diffusion carriers and to increase the drift carriers a lower doping regions and a large bias voltage is necessary to create a wider space charge region with strong electric field. As the doping increases the mobility decreases and the diffusion time increases

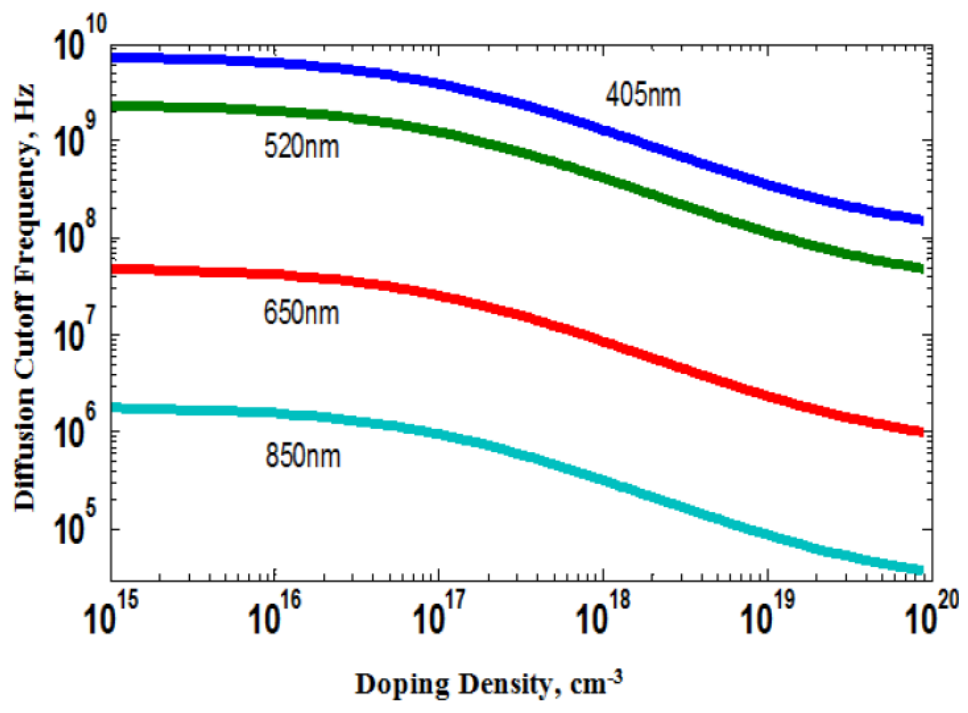

Fig. 3: Electron diffusion cutoff frequency (intrinsic bandwidth) as a function of the reverse biasing.

leading to smaller intrinsic bandwidth. The carrier diffusion should be reduced to obtain high speed photodiodes. One possibility is to use light with a short wavelength (large absorption coefficient) to avoid photogeneration in the substrate (region 2). A larger reverse voltage should be applied across the photodiode or the doping concentration in the photodiode should be reduced, in order to obtain a thicker space-charge region to reduce the diffused carriers and increase the drift carriers' contributions. Different photodiode structures like spatially modulated photodiode (SML)[5], fingered photodiode[6], PIN-PD with Pepi layer [7], and photodiode with body contacts 
[8] are used to increase the photodiode speed by reducing the diffusion current. The extrinsic bandwidth of the photodiode due to its capacitance is determined by the input resistance of the next stage (TIA), see Figure 2.

$$
B W_{e X}=\frac{1}{2 \pi R_{i n} C_{P D}}
$$

The total bandwidth of the photodiode can be calculated by:

$$
B W_{\text {total }}^{-2}=B W_{e x}^{-2}+B W_{\text {in }}^{-2}
$$

Figure 4 the junction capacitance as a function of the reverse biasing volt for different substrate doping level. Decreasing the substrate doping or increasing the biasing volt will increase the junction width which has the effect of decreasing the junction capacitance. The extrinsic cut off frequency of the photodiode due to its capacitance is determined by the input resistance of the next stage (TIA), see Figure 2.

The influence of carrier diffusion on the frequency response of photodetectors is shown in Figure 5. The total intrinsic photodiode frequency response in Figure 5 shows a small roll-off $(5 \mathrm{~dB})$ due to the combination of the drift and diffusion photocurrent components. The total photocurrent response roll-off is smaller than the diffusion roll-off in region 2, due to the larger influence of the faster response of diffusion region 1 and the drift region currents.

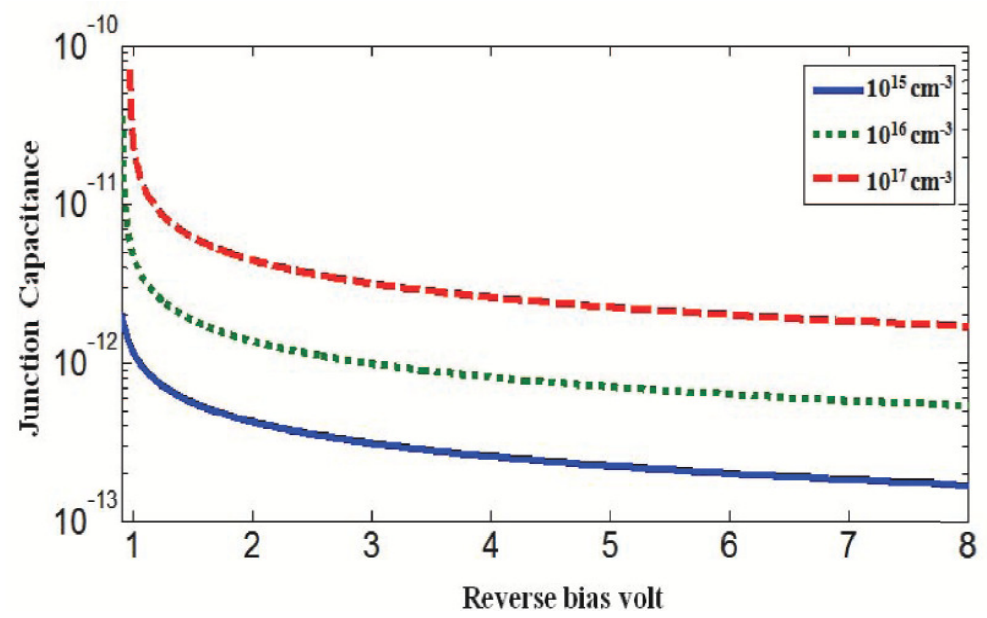

Fig. 4: The junction capacitance as a function of the reverse biasing volt for different substrate doping level. 


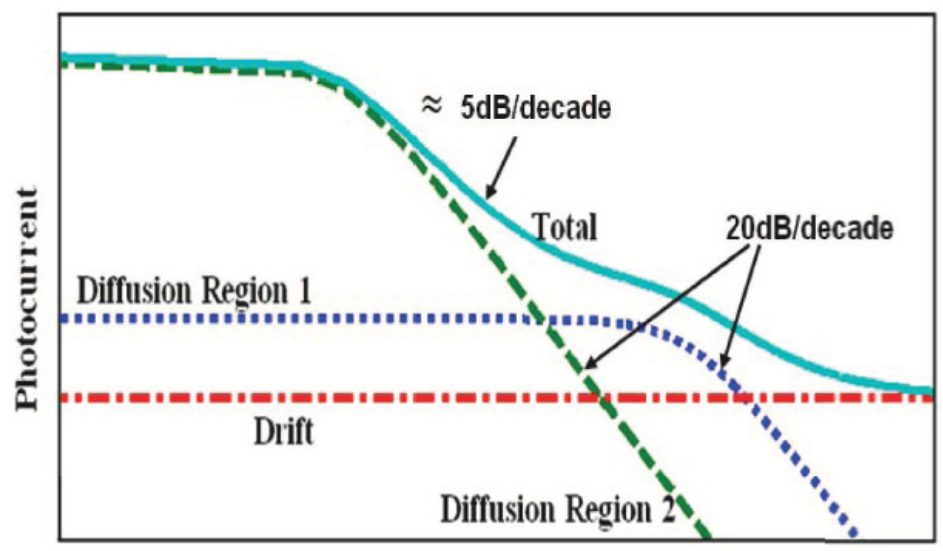

Frequency

Fig. 5: Frequency response of the photodiode photocurrent considering drift and diffusion components.

\section{Optical Quantum Efficiency and Responsivity}

\subsection{Quantum Efficiency}

The reflection behavior at integrated CMOS photodiode surface layers is more difficult to evaluate since there are several layers involved. The situation gets worse in each newer generation of CMOS technology, because the number of metal and dielectric layers increases. In addition to these dielectric layers, there are two passivation layers on top of the last metal layer. This makes the optical behavior by far more critical since the variation of transmission $\mathrm{T}$ over wavelength becomes significantly higher.

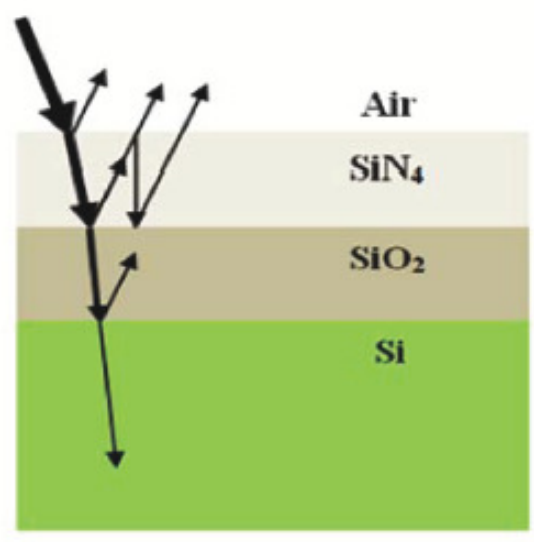

Fig. 6: Transmission and reflection of an incident light at typical CMOS photodiode Nitride and Oxide surface layers.

In almost every CMOS technology the uppermost layer is silicon nitride $\left(\mathrm{Si}_{3} \mathrm{~N}_{4}\right)$ for passivation that protects the fabricated devices from environmental influences. Below the nitride layer there are different oxide layers $\left(\mathrm{SiO}_{2}\right)$ used as inter -metal - oxide to isolate the metal lines. A typical layer combination for such photodiodes is drawn in Figure 6. 
The light reflection $\mathrm{R}_{\mathrm{opt}}$ only depends on the refractive index $\mathrm{n}$ of the two different layers [9]:

$$
R_{o p t}=\frac{\left(n_{1}-m_{2}\right)^{2}}{\left(n_{1}+m_{2}\right)^{2}}
$$

Since there is a certain amount of reflection on every layer interface, the superposition of multiple reflected waves with the incident wave must be considered. The phase shift of a wave entering a layer from topside and reflected back at bottom side interface is given by [10]:

$$
\sigma=\frac{4 \pi}{\lambda} n_{i} d_{i}
$$

Depending on layer thickness $d_{i}$ and refractive index ni the superposition of all reflected wave contributions shows minimum and maximum at certain wavelengths $\lambda$. To calculate the $R_{\text {opt }}$ of multiple layer structures, different methods were developed by using matrix algebra mathematics [10].

The simulated transmission coefficient for different wavelengths is plotted in Fig. 7.

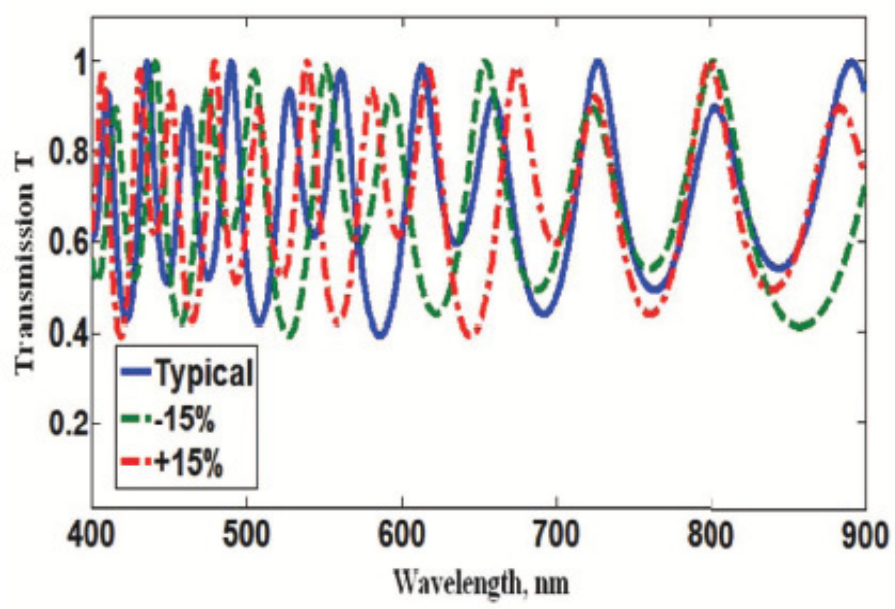

Fig. 7: Light transmission at a typical CMOS surface layer stack with nitride and oxide on silicon

The spectral behaviour shows a strong variation of transmission coefficient $\left(T=1-R_{\text {opt }}\right)$ over wavelength from very high values of $1(=100 \%)$ down to low values of $0.4(=40 \%)$. This can be explained by the refractive indices which changes from $n=1$ for air to a high value of $n_{\text {sn }}=2.5$ for nitride, back to a lower value $n_{o x}=1.46$ for oxide and again to a high value of $n_{s i}=4.18$ for silicon. To improve the transmission, the refractive indexes should increase continuously from one layer to another. So the nitride layer has to be removed from top and instead inserted between oxide and silicon. A risk by doing this is the missing passivation surface. So humidity might permeate into the silicon substrate and change the transistors behavior over lifetime. This effect has to be investigated during photodiode qualification process.

The nitride layer with defined thickness can be used as an anti-reflection-coating (ARC) on the photodiode and can improve the light transmission. The drawback is that additional process steps are necessary resulting in a non-standard IC process flow with higher production costs. The reflection coefficient can be minimized with an ARC at the surface of the semiconductor for a 
certain wavelength or a more or less small wavelength range. The optimum values of the thickness of the ARC $\mathrm{d}_{\mathrm{ARC}}$ and its refractive index $\mathrm{n}_{\mathrm{ARC}}$ can be calculated with the formulas 3.37 and 3.38 with the refractive index of the surrounding $\mathrm{nS}$ and the refractive index of the semiconductor $\mathrm{n}_{\mathrm{SC}}$. The materials $\mathrm{SiO} 2\left(\mathrm{n}_{\mathrm{ARC}}=1.45\right)$ and $\mathrm{Si} 3 \mathrm{~N} 4\left(\mathrm{n}_{\mathrm{ARC}}=2.0\right)$ are most adequate for the ARC coating.

$$
\begin{array}{r}
n_{A R C}=\sqrt{n_{S} n_{S C}} \\
d_{A R C}=\frac{\lambda_{0}}{4 n_{A R C}}
\end{array}
$$

\subsection{Responsivity}

The conversion factor from optical power POPT to electrical current IPH is defined by the responsivity $\mathrm{R}$, it can be calculated by:

$$
R=\frac{I_{P H}}{P_{O P T}}=\frac{\eta \lambda[\mathrm{nm}]}{1240}
$$

Figure 8 shows the photodiode responsivity calculated $\mathrm{R}$ after considering the optical efficiency and internal quantum efficiency section 4.1.

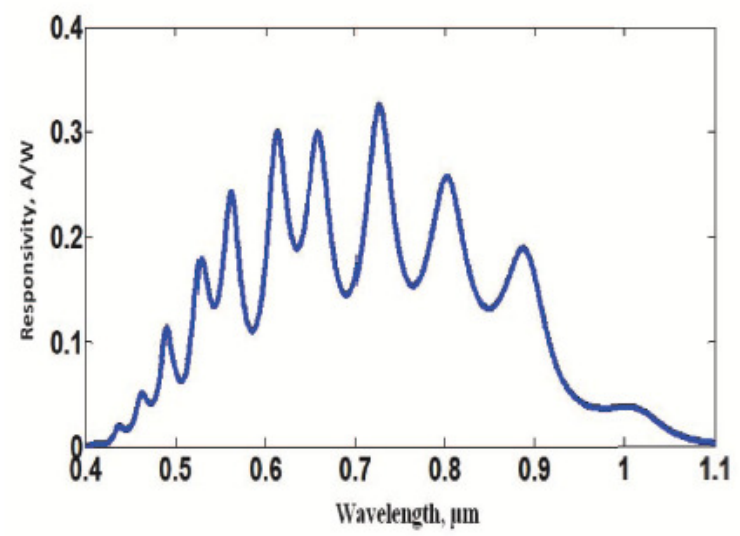

Fig. 8: Responsivity of silicon photodiode as a function of wavelength after considering the optical efficiency 


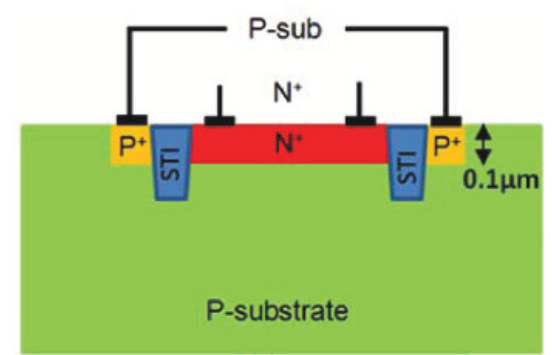

(a)

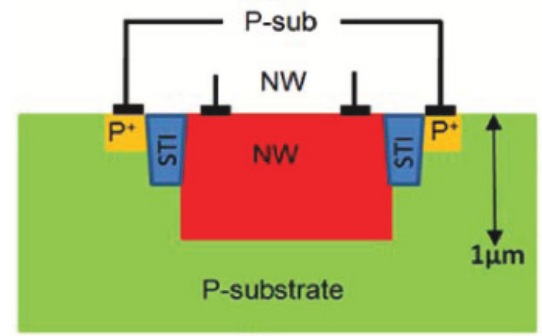

(b)

Fig. 9: Photodiode structures (a) N+/P-substrate, (b) NW/P-substrate

For short wavelengths the responsivity goes to zero close to the silicon surface because of surface recombination. Surface recombination is due to surface states that come from the crystal imperfection and the oxidized silicon that grows at the surface of silicon.

Also, the short wavelengths responsivity decreases due to recombination when the junction distance from the surface (junction depth) increases. Long wavelengths responsivity decrease if the recombination in the substrate increases. Responsivity increases, for long wavelengths, with increasing SCR width. The strong fluctuation in the responsivity due to reflectance from dielectrics stack in 90nm CMOS process was also reported in [11]. A simulation analysis for the optical transmission coefficient in $130 \mathrm{~nm}$ CMOS process and its effect on the responsivity was introduced in $[12,13]$.

\section{CMOS Photodiodes Structures}

\subsection{Classical PN Junctions}

The simplest way to build CMOS photodiodes is to use the classical PN junctions available in CMOS processes: source or drain/substrate, and well/substrate diodes. Figure 9 shows the structure of the proposed PDs fabricated with a standard 40nm CMOS process [14]. In Fig. 9(a) the $\mathrm{N}+$ source/drain implant with the P-substrate are used to implement the PN photodiode. In Fig. 9(b) the $\mathrm{N}$-well with the P-substrate implement another PN photodiode structure. Due to the shallow trench isolation (STI), the breakdown voltages of the N+/P-substrate photodiode is $18.5 \mathrm{~V}$ and the breakdown voltage of the NW/P-substrate photodiode is $16.3 \mathrm{~V}$. The STI prevents premature edge breakdown for N+/P-substrate PD but cannot do this for the NW/P-substrate PD because the NW is deeper than the STI. Therefore, the maximum bias voltages of $18 \mathrm{~V}$ and $14.8 \mathrm{~V}$ were chosen for the $\mathrm{N}+/ \mathrm{P}$-substrate and the NW/P-substrate photodiode, respectively. The leakage currents for these diodes with an area of $70 \mu \mathrm{mx} 70 \mu \mathrm{m}$ were $50 \mathrm{nA}$ at these bias voltages. Each one 
of the two PDs is designed with different dimensions $30 \mu \mathrm{mx} 30 \mu \mathrm{m}, 50 \mu \mathrm{mx} 50 \mu \mathrm{m}$ and $70 \mu \mathrm{mx} 70 \mu \mathrm{m}$.

The responsivity of the presented PDs can be increased to $0.16 \mathrm{~A} / \mathrm{W}$ by increasing the reverse bias voltages more than $18 \mathrm{~V}$ for the $\mathrm{N}+/ \mathrm{P}$-substrate, and to $0.38 \mathrm{~A} / \mathrm{W}$ at $14.8 \mathrm{~V}$ for NW/P-substrate $\mathrm{PD}$ to induce avalanche multiplication but at the expense of larger leakage current and larger excess noise [5].

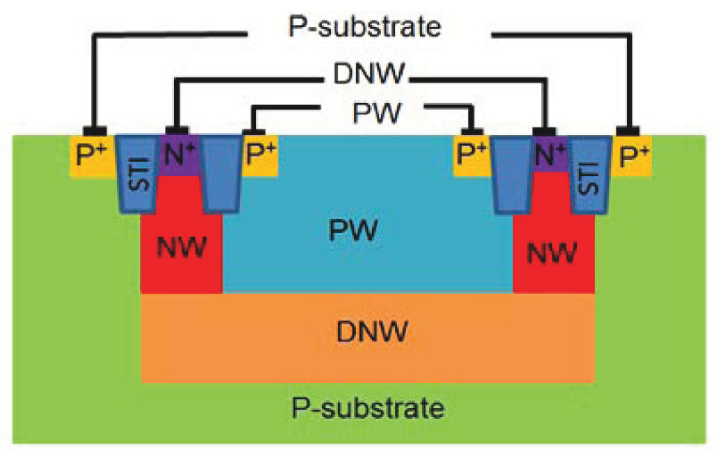

Fig. 10: DPD PW/DNW/P-substrate structure

The measured N+/P-substrate and the NW/P-substrate PDs show comparable capacitance values. As the capacitance of the NW/P-substrate PD goes from $0.04 \mathrm{pF}$ at $30 \mu \mathrm{mx} 30 \mu \mathrm{m}$, via $0.12 \mathrm{pF}$ for $50 \mu \mathrm{m} \times 50 \mu \mathrm{m}$ to $0.21 \mathrm{pF}$ for $70 \mu \mathrm{m} \times 70 \mu \mathrm{m}$ at $8 \mathrm{~V}$.

The frequency response of the $30 \mu \mathrm{mx} 30 \mu \mathrm{m} \mathrm{N}+/ \mathrm{P}-$ Substrate PD for $850 \mathrm{~nm}$ shows a very small bandwidth $(40 \mathrm{MHz}$ at $18 \mathrm{~V})$ because of the larger diffusion current. The $\mathrm{N}+$ region is very shallow $(0.1 \mu \mathrm{m})$ and most of the photons for the $850 \mathrm{~nm}$ are absorbed deep in the substrate that is far away from the junction. Even with the high bias voltage of $18 \mathrm{~V}$ (width of the space-charge region is $4.7 \mu \mathrm{m})$ carrier diffusion still dominates. The frequency response for the $30 \mu \mathrm{mx} 30 \mu \mathrm{m}$ NW/PSubstrate PD is introduced for $850 \mathrm{~nm}$ has a bandwidth of $40 \mathrm{MHz}$ at $14.8 \mathrm{~V}$, which is rather small because of the large diffusion current. The NW's depth is around $1 \mu \mathrm{m}$ and most of the $850 \mathrm{~nm}$ light is absorbed in the substrate that is far away from the junction. Even for the high bias voltage of $14.8 \mathrm{~V}$ the diffusion current still dominates over the drift current.

\subsection{PW/DNW/P-Substrate Double Photodiode (DPD)}

A PW/DNW/P-substrate DPD was implemented in 40nm CMOS technology [16]. Fig. 10 shows the structure of the PW/DNW/P-substrate DPD. The DPD is formed by PW/DNW and DNW/Psubstrate junctions. The breakdown voltage of the PW/DNW/P-substrate DPD is $15.22 \mathrm{~V}$. The bias voltage of $15.1 \mathrm{~V}$ was chosen to achieve a maximum responsivity. The leakage current for the PW/DNW/P-substrate DPD with an area of $70 \mu \mathrm{mx} 70 \mu \mathrm{m}$ at these voltages was 100nA.

The DPD's capacitance is the sum of the capacitances of two parallel junctions. The capacitance of the $70 \mu \mathrm{mx} 70 \mu \mathrm{m}$ PW/DNW/P-substrate DPD is $1.9 \mathrm{pF}$ at $8 \mathrm{~V}$. The frequency response for the $70 \mu \mathrm{mx} 70 \mu \mathrm{m}$ PW/DNW/P-Substrate DPD is measured for $850 \mathrm{~nm}$. The bandwidth is $4 \mathrm{MHz}$ at $1 \mathrm{~V}$, which is rather small because of the large diffusion current. The PW's depth is around $1 \mu \mathrm{m}$ and most of the $850 \mathrm{~nm}$ light is absorbed in the substrate far away from the junction. Even with the high bias voltage of $15.1 \mathrm{~V}$ the bandwidth increased a little bit to $9 \mathrm{MHz}$ because the diffusion current still dominates over the drift current. 


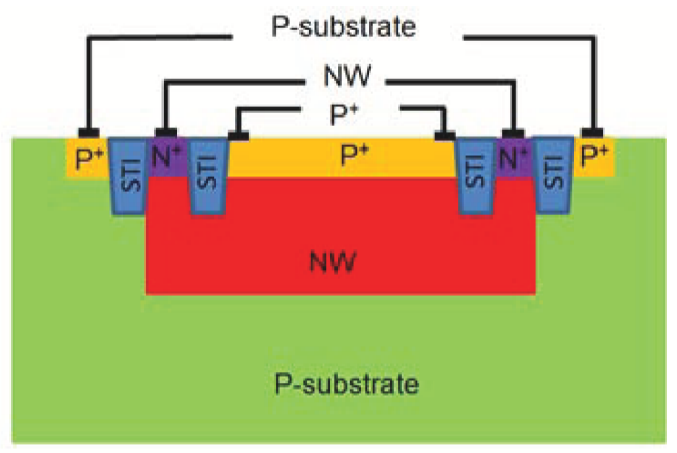

Fig. 11: $\mathrm{P}+/ \mathrm{NW} / \mathrm{P}-$ substrate DPD structure.

\subsection{P+/NW/P-Sub Avalanche Double Photodiode}

Figure 11 shows the structure of a P+/NW/P-substrate DPD. A standard 40nm CMOS process was used in this design without any process modifications. The $\mathrm{P}+$ source/drain implants with $\mathrm{NW}$, and the P-substrate are used to implement the avalanche double photodiode (ADPD) [16]. The basic structure of the presented ADPD is formed by $\mathrm{P}+/ \mathrm{NW}$ and NW/P-substrate junctions where the avalanche effect occurs at the $\mathrm{P}+/ \mathrm{NW}$ junction. The breakdown voltage of the $\mathrm{P}+/ \mathrm{NW}$ junction is $8.44 \mathrm{~V}$. The shallow trench isolation (STI), prevents the premature edge breakdown for the $\mathrm{P}+/ \mathrm{NW}$ junction but cannot prevent it for the PW/DNW junction because the PW is deeper than the STI (Fig. 9). In principle, this ADPD can be connected to the amplifier in two ways: first, the NW cathode can be used as output and the P+ anode can be connected to a negative bias voltage and second, the $\mathrm{P}+$ anode can be connected to the amplifier input and the NW cathode can be biased by a positive voltage. But, the second way should be preferred because slow diffusion currents from the substrate do not contribute. For the measurements, the bias voltage of $8.41 \mathrm{~V}$ was chosen for the P+/NWADPD, to achieve a maximum responsivity. The leakage current for the $\mathrm{P}+/ \mathrm{NW} / \mathrm{P}$-substrate ADPD, with an area of $70 \mu \mathrm{mx} 70 \mu \mathrm{m}$, is $3 \mu \mathrm{A}$ at $8.41 \mathrm{~V}$.

By increasing the bias voltage the responsivity increases due to the widening of the space-charge regions and more drift current will be collected. The large increase in the $\mathrm{P}+/ \mathrm{NW} / \mathrm{P}$-substrate PD's responsivity is because of the avalanche effect which occurs at the $\mathrm{P}+\mathrm{NW}$ junction. The STI prevents the premature edge breakdown for the $\mathrm{P}+\mathrm{NW}$ junction. The short wavelength have higher responsivity than the long wavelengths. The short wavelengths are strongly absorbed in the $\mathrm{p}+$ region. Since all photons are absorbed before the multiplication region; short wavelengths have a pure electron injection in the multiplication region. For long wavelengths electron-hole pairs generated beyond the multiplication region, only holes reach the multiplication region. The lower gain of long wavelengths results from the lower ionization coefficient of holes than electrons, so they undergo a reduced gain. To measure the total responsivity for the P+/NW/P-sub DPD, the substrate is shorted with the $\mathrm{P}+$. A $127 \mu \mathrm{W} 850 \mathrm{~nm}$ vertical cavity surface emitting laser (VCSEL) was used. An avalanche multiplication occurs in the P+/NW/P-sub DPD's responsivity and peak responsivities of $0.84 \mathrm{~A} / \mathrm{W}$ were achieved for $850 \mathrm{~nm}$ where the unity-gain responsivities was $0.076 \mathrm{~A} / \mathrm{W}$ for $850 \mathrm{~nm}$.

The measured capacitance is $3.12 \mathrm{pF}$ for the $\mathrm{P}+/ \mathrm{NW} / \mathrm{P}$-substrate DPD at $8 \mathrm{~V}$. The $\mathrm{P}+\mathrm{NW} / \mathrm{P}$ substrate diode has a higher capacitance than the $\mathrm{PW} / \mathrm{DNW} / \mathrm{P}$-substrate. The higher $\mathrm{P}+$ doping concentration compared to the PW doping leads to a thinner depletion region and higher capacitance for the $\mathrm{P}+/ \mathrm{NW}$ junction compared to the PW/DNW junction [13]

$\mathrm{P}+/ \mathrm{NW} / \mathrm{P}$-substrate DPD introduced in Fig. 11 for $850 \mathrm{~nm}$ shows a very small bandwidth (3MHz at $1 \mathrm{~V})$ because of the large diffusion current. The $\mathrm{P}+$ region is very shallow $(0.1 \mu \mathrm{m})$ and most of the 
photons for the $850 \mathrm{~nm}$ are absorbed deep in the substrate far away from the junction. For higher bias voltages the bandwidth increases due to the wider space-charge region and the higher electric field in the two $\mathrm{P}+/ \mathrm{NW}$ and NW/P-substrate junctions. At $8.44 \mathrm{~V}$ bias the bandwidth increased to $700 \mathrm{MHz}$.

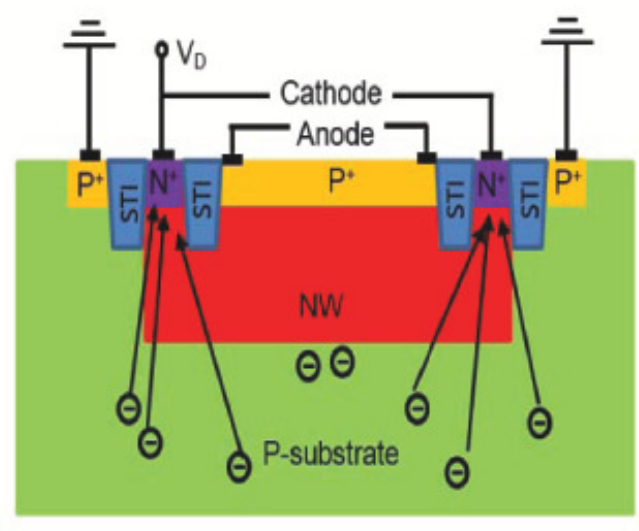

Fig. 12: Structure of $\mathrm{P}+/ \mathrm{NW} / \mathrm{P}$-substrate photodiode with guard ring.

\subsection{P+/NW/P-Substrate Photodiode with Guard}

The two DPDs introduced in the previous sections 4. 2 and $\mathrm{C}$ have a high capacitance; since the DPD has two junctions and the total capacitance is the sum of the two junction capacitances. Also the substrate diffusion current contributes to the total photocurrent; slowing down the PD speed. Another technique can be used to reduce the PD capacitance and to block the substrate diffusion current. The double junction $\mathrm{PD}$ is a $\mathrm{P}+/ \mathrm{NW} / \mathrm{P}-$ sub with a $\mathrm{P}+/ \mathrm{NW}$ junction together with the NW/P-sub junction. The structure of $\mathrm{P}+/ \mathrm{NW} / \mathrm{P}$-substrate photodiode with guard ring is shown in Figure 12 (a). $\mathrm{P}+/ \mathrm{NW}$ junction is used to detect the optical signal, and the $\mathrm{P}$-sub/N-well junction acts as guard (equalizing PD) collecting the slowly diffusing carriers generated in the substrate $[18,2]$.

The NW cathode current consists of the electrons diffusion current from the substrate as well as of a part of the drift component of the depletion region, while the $\mathrm{P}+$ anode current consists of a part of the diffusing holes from the NW and of a drift current. Most of the diffusing electrons coming from the substrate will be collected by the cathode (NW). By connecting the NW cathode to a high positive voltage $\mathrm{VD}$, while connecting the anode $(\mathrm{P}+)$ to the subsequent amplifier, maximum speed can be achieved. In other words, the P-sub/N-Well junction collects most of the diffusing photocarriers, shielding the upper photodiode junction $\mathrm{P}+/ \mathrm{N}-\mathrm{Well}$ from these slow components. This technique increases the PD's speed, but on the expense of smaller responsivity. The capacitance of the $\mathrm{P}+/ \mathrm{NW} / \mathrm{P}$-sub with guard is only the capacitance of the $\mathrm{P}+/ \mathrm{NW}$ junction, which is smaller than the DPD capacitance.

Simulation results at $600 \mathrm{~nm}$ and $850 \mathrm{~nm}$ for a double junction PD with a dimension of $65 \mu \mathrm{mx} 65 \mu \mathrm{m}$ in $130 \mathrm{~nm}$ CMOS with guard were presented in [13]. The PD has a bandwidth of 790 $\mathrm{MHz}$ in response to $850 \mathrm{~nm}$ light and $624 \mathrm{MHz}$ for $600 \mathrm{~nm}$. The bandwidth of the double junction photodiode with guard is higher than the simple PN junction's bandwidth (and the DPD bandwidth in previous sections and the responsivity of the double junction photodiode with guard is less than that of the simple PN junction responsivity. This is due to shielding of the substrate current component in the double junction photodiode with guard. The double junction photodiode responsivity is $74.8 \mathrm{~mA} / \mathrm{W}$ at $600 \mathrm{~nm}$ and $17.6 \mathrm{~mA} / \mathrm{W}$ for $850 \mathrm{~nm}$. For shorter wavelengths $(600 \mathrm{~nm})$, the responsivity is higher than at $850 \mathrm{~nm}$ wavelength. The reason is that for shorter wavelengths, the penetration depth of light is smaller and thus the percentage of substrate current is less. 
Therefore, less current is absorbed by the cathode and more current is available at short wavelengths. A P+/NW/P-sub APD with guard was fabricated in $130 \mathrm{~nm}$ standard CMOS technology [8]. The PD is formed from the $\mathrm{P}+\mathrm{NW}$ junction with the $\mathrm{P}+$ contacts for the substrate are tied to ground. In order to be able to exploit the avalanche effect, VD has to be much larger than Vdd. An optical window having the area of $30 \mu \mathrm{m} \times 30 \mu \mathrm{m}$ was formed by blocking the salicide process. The total device area was $43.8 \mu \mathrm{m} \times 43.8 \mu \mathrm{m}$. The photodetector was realized without violating any design rule for the CMOS process technology. For characterization of the APD, an $850 \mathrm{~nm}$ laser source was used. The avalanche breakdown voltage was about $9.8 \mathrm{~V}$ and the peak responsivity was $20.3 \mathrm{~A} / \mathrm{W}$. The PD had a $3-\mathrm{dB}$ bandwidth of $2.5 \mathrm{GHz}$, which is higher than that of the DPD introduced in previous sections; due to the effect of the equalizing PD and the lower doping in $130 \mathrm{~nm}$ technology compared to 40nmCMOS technology.

\subsection{Fingered Photodiodes}

The short wavelengths (blue light) have low quantum efficiency. The majority of the photogenerated carriers, therefore, will strongly recombine near the surface and in the $\mathrm{N}+$ region. The carriers will diffuse slowly due to the low electron and hole mobilities in the highly doped $\mathrm{N}+$ region. The slow carrier diffusion processes outside the space-charge region are responsible for a limited response speed. To reduce both, recombination and diffusion, space-charge regions at the surface have to be implemented. An interdigitated network of N+ fingers is employed instead of a continuous $\mathrm{N}+$ region for maximizing the depletion regions available for carrier collection, particularly near the surface of the device. An interdigitated structure is shown in Fig. 13. The Psub PD is used as the anode and the $\mathrm{N}+$ fingers are used as the cathode of the photodiode. The $\mathrm{N}+$ fingers are connected by metal outside of the photo-sensitive region. Photogenerated carriers can be separated in the space-charge region, electrons drift to the $\mathrm{N}+$ fingers and holes drift to the Psub. For a small number of fingers the distance between fingers is so wide that carrier diffusion should be expected to contribute and to slow down the response speed. The photodiode speed can be increased by increasing the number of fingers.

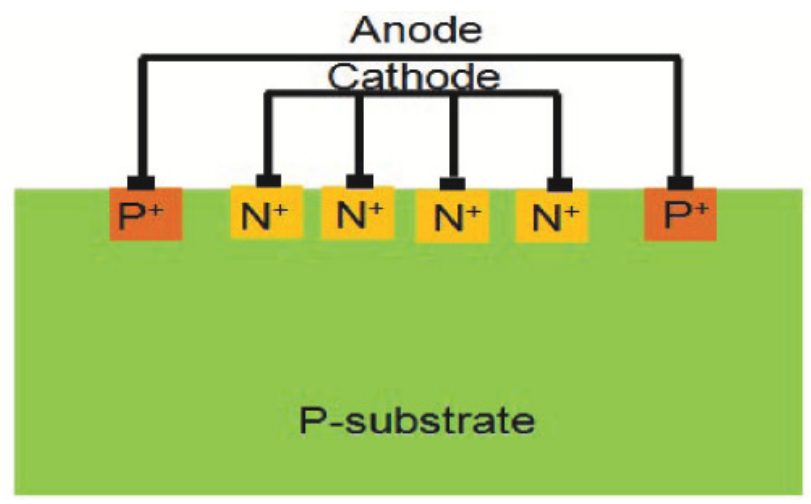

Fig. 13: Cross section of an interdigitated finger N+/P-sub photodiode. 


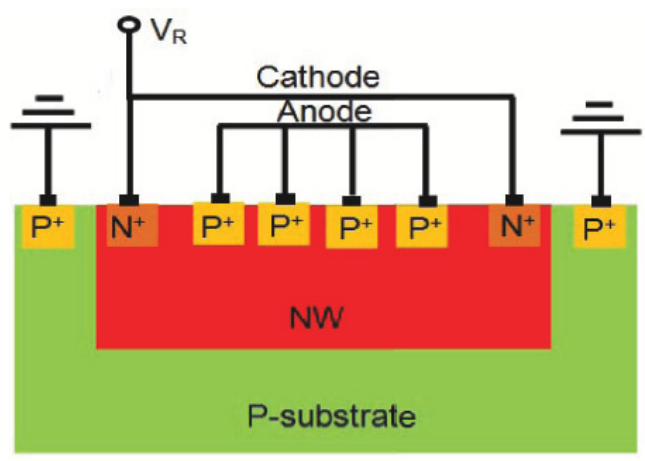

Fig. 14: Cross section of an interdigitated finger $\mathrm{P}+/ \mathrm{NW} / \mathrm{P}-$ sub photodiode

The space charge regions of neighboring $\mathrm{N}+$ regions overlap and there is no carrier diffusion (outside the $\mathrm{N}+$ regions) involved for blue light. A $\mathrm{P}+\mathrm{NW} / \mathrm{P}$-sub interrupted $\mathrm{P}+$ finger photodiode fabricated in $0.35 \mu \mathrm{m}$ CMOS process, was introduced in $[19,20]$, see Fig. 14. The P+/NW junction forms the PD. The slow diffusion of carriers generated by light with a wavelength of $850 \mathrm{~nm}$ in the P-sub, therefore, does not contribute to the photocurrent, if the NW cathode is connected to a (positive) supply voltage and the anode is connected to the amplifier input. This slow current is collected by the NW and shorted to the power supply. To enhance the speed of the photodiode, an interdigitated network of $\mathrm{P}+$ fingers is employed instead of a continuous $\mathrm{P}+$ region for maximizing the depletion regions available for carrier collection, particularly near the surface of the device. The NW had a size of $16.5 \mu \mathrm{mx} 16.5 \mu \mathrm{m}$. With a detector bias of $10 \mathrm{~V}$ a bit rate of $1 \mathrm{Gbit} / \mathrm{s}$ was reported. The responsivity of the photodiode with the reported values of $0.01-0.04 \mathrm{~A} / \mathrm{W}$ was very low due to the shallow $\mathrm{P}+\mathrm{NW}$ junction.

In [21], a PD with multiple PW/NW structure in standard 180nm CMOS technology has been presented. The PW together with the P-sub and the NW form the avalanche structure, see Figure 15. In addition, the standard shallow trench isolation (STI) is also implemented in between wells to reduce surface leakage and enhance breakdown characteristics. The PW is deeper than the NW and the PW is higher doped than the NW. The total active area of this PD is $50 \mu \mathrm{mx} 50 \mu \mathrm{m}$, which consists of seven PN diodes. For each diode, the width for PW(WP), NW(WN), STI oxide (WSTI), and contact metal strip is $1.95,3.45,0.36$, and $0.45 \mu \mathrm{m}$, respectively. The distance that the generated carriers drift is determined by the width of each diode and thus affects the drift time directly.

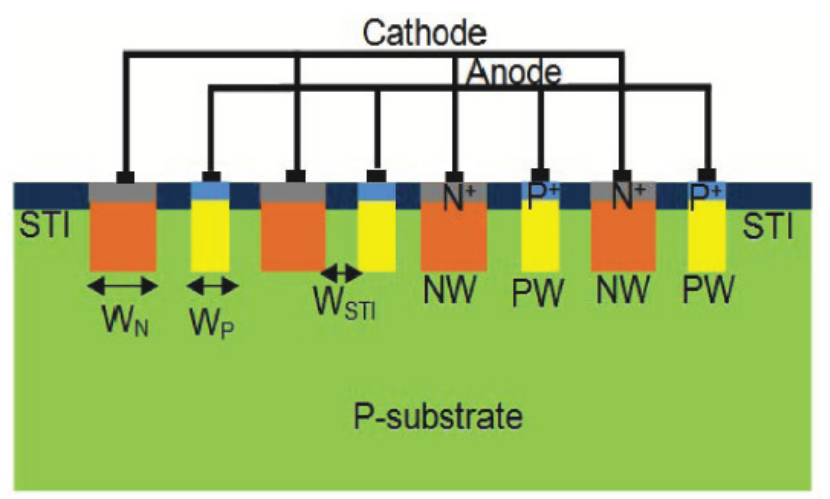

Fig. 15: Cross section of an interdigitated finger PW/NW/P-sub photodiode 
In this design, the width of each well and STI oxide is minimized and limited by design rules. At suitable reverse bias, the NW and P-sub region are depleted and converted into a wide absorption region. By using STI oxide, it not only improves the breakdown voltage but also extends the depletion region. Therefore, the fully depleted region is wider than published Si PD using P-sub as P-region only. Accordingly, it would improve the responsivity. The PD shows a high responsivity of $0.37 \mathrm{~A} / \mathrm{W}$ at zero bias. At a reverse bias of $14.3 \mathrm{~V}, \mathrm{PD}$ demonstrates a high responsivity of 0.74 $\mathrm{A} / \mathrm{W}$, a $3-\mathrm{dB}$ bandwidth of $1.6 \mathrm{GHz}$.

\subsection{PIN Photodiode}

A PIN-PD consists of a P (anode) and N (cathode) with an intrinsic (I) layer in between. The PIN$\mathrm{PD}$ is reverse biased, to achieve a strong electric field in the intrinsic layer. Electron-hole pairs photogenerated in the intrinsic region, are immediately separated by the high electric field in the intrinsic region. The internal efficiency and the speed of the PIN-PD depend on the intrinsic region width. The wider the intrinsic region is, the higher is the internal efficiency. Also, the PD's capacitance is decreasing by increasing the intrinsic region's width and a higher bandwidth can be obtained. On the other hand, the wider the intrinsic region's width is, the longer the electrons and holes need to drift through the intrinsic region. Therefore a tradeoff between efficiency and speed should be found. The P- epi-layer, which has lower doping than the heavily doped substrate, is used to pair with NW to form the intrinsic region (I) in a vertical PIN PD, Fig. 16. In such a PINPD structure, the electric field is largely confined inside the P- epi-layer, between the P+ substrate (Anode) and $\mathrm{N}+$ region (cathode).

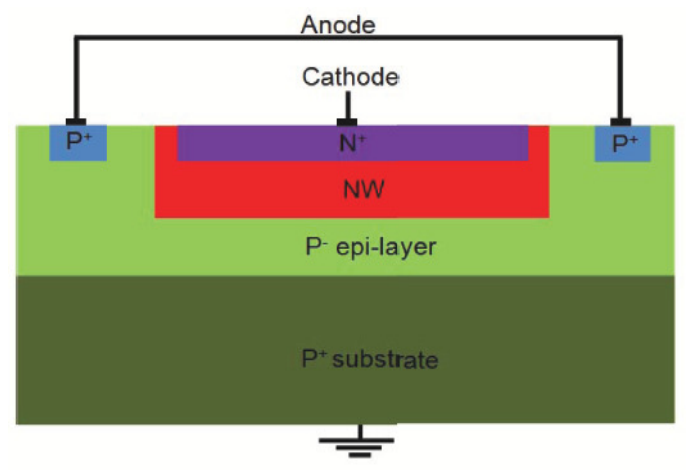

Fig. 16: Cross section of a vertical PIN photodiode [7].

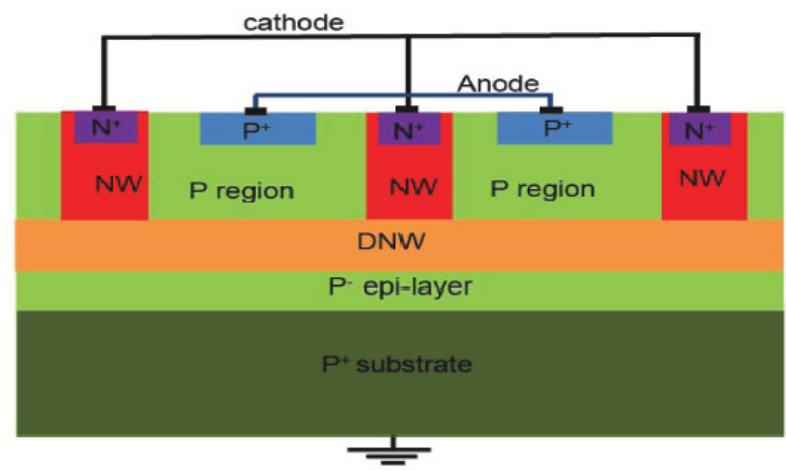

Fig. 17: Cross section of PIN photodiode with DNW [7]. 
One advantage of epi-CMOS PDs is that when the epi-layer is thin enough, a low bias voltage can deplete the entire epi-layer, because of its low doping level. Second advantage, the minority carrier recombination is faster in the $\mathrm{P}+$ substrate than in the bulk CMOS process, and hence most photogenerated electrons inside the substrate recombine during diffusion before reaching the depletion region. This will reduce the diffusion current effect and a faster PD is expected but at lower responsivity. A $120 \mu \mathrm{mx} 120 \mu \mathrm{m}$ vertical PIN-PD was fabricated in $180 \mathrm{~nm}$ CMOS technology with epi-layer. The vertical PIN-PD showed a responsivity of $0.135 \mathrm{~A} / \mathrm{W}, 0.77 \mathrm{pF}$ capacitance, and a bandwidth of $1.15 \mathrm{GHz}$ at $5 \mathrm{~V}$ reverse bias and for $850 \mathrm{~nm}$ light. At $15.5 \mathrm{~V}$ the bandwidth increased to $1.61 \mathrm{GHz}[7]$.

A kind of PIN-PD structure can be implemented by adding inside a DNW a PW region as shown in Fig. 17 [7]. The DNW forms the cathode of the upper vertical PIN P+/PW/DNW PD. The NW is the cathode of the lateral P+/PW/NW PIN PD. The bottom DNW/P-epi/P-sub junction, with the substrate connected to ground, can be used to isolate the PIN PD from the substrate and to block the substrate diffusion current. The PD structure with the DNW combines the advantages of vertical and lateral PIN devices to enhance the electric field inside the epi-layer. Therefore, it can improve the PD's bandwidth without sacrificing too much responsivity. A DNW PIN-PD with an area of $70 \mu x m 70 \mu \mathrm{m}$ achieved a $3 \mathrm{~dB}$ bandwidth of more than $3.13 \mathrm{GHz}$ at $15.5 \mathrm{~V}$ bias. At $5 \mathrm{~V}$ bias, the same DNW PD achieved $2.19 \mathrm{GHz}$ bandwidth, versus about $1.15 \mathrm{GHz}$ for a conventional vertical PIN PD [7]. At $850 \mathrm{~nm}$, its responsivity was measured to be $0.14 \mathrm{~A} / \mathrm{W}$ corresponding to a quantum efficiency of $20 \%$ when biased below $10 \mathrm{~V}$. The responsivity increased to $0.4 \mathrm{~A} / \mathrm{W}$ or a quantum efficiency of $58 \%$ at $16.2 \mathrm{~V}$ bias. Its dark current was $0.46 \mathrm{nA}$ at $5 \mathrm{~V}$ bias [7].

\subsection{Spatially Modulated Light Detector}

The spatially modulated light (SML) detector was first introduced in [22] to increase the PD speed by subtracting a part of the diffusion current. Fig. 18 shows the cross section of the SML detector. Half of the fingers are shielded from the incident light by floating metal strips. The shielded fingers connected together constitute the "deferred detector". The other unshielded fingers connected together constitute the "immediate detector". When light is incident on the detector, the metal mask blocks the incident light on the deferred detector, and light is absorbed in the immediate detector. Photocarriers are generated below the immediate detector region and not under the deferred region. The incident light is spatially modulated according to the metal covered and uncovered regions. The carriers generated close to an illuminated junction will have a higher probability to be collected by the immediate detector junctions. Bulk generated carriers (diffusion) have the same probability to reach the immediate or differed detector junctions. The deferred current (slow diffusion only) is subtracted from the immediate current (both slow diffusion and fast drift components), to obtain the "effective detector" current. The effective detector current has a faster response; because part of the slow diffusive carriers is cancelled by the subtraction.

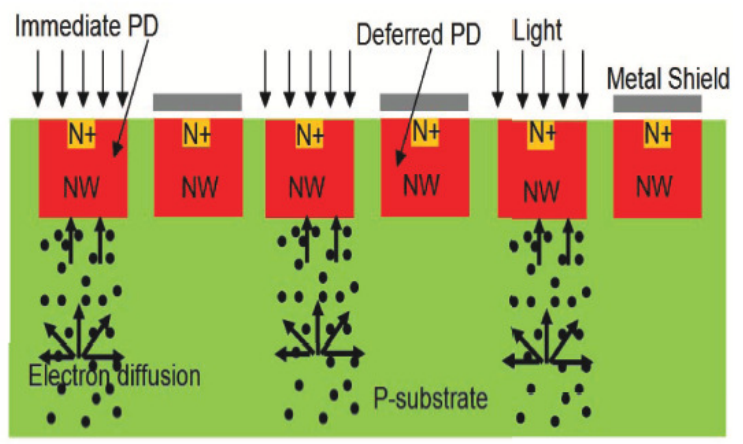

Fig. 18: Cross section of spatially modulated light detector. 
The diffusion component in the P-sub determines the SML photodiode bandwidth. The smaller the distance between the adjacent NWs is, the better the diffusion cancellation works, and the higher the bandwidth is [23]. The speed is improved on the expense of a lower responsivity. Half of the light is reflected from the shielded fingers, and more deferred detector current is subtracted from the immediate detec tor current by increasing the number of fingers. The SML detector has a smaller capacitance compared to a reference PD, without using SML, because the SML is split into two PDs. The receiver sensitivity increases somewhat due to the low SML detector's capacitance decreasing the input capacitance. The low responsivity of the SML detector is partially compensated by the lower capacitance of the device.

The subtraction is performed by connecting the SML PDs to a differential transimpedance amplifier (TIA). The differential TIA has the advantages of higher rejection of supply noise, and better linearity due to the suppression of even harmonics compared to a single ended TIA.

The N+/NW/P-Sub SML photodetector introduced in [24], fabricated in $0.8 \mu \mathrm{m}$ CMOS, has a DC responsivity of $0.05 \mathrm{~A} / \mathrm{W}$ at $860 \mathrm{~nm}$ and $0.132 \mathrm{~A} / \mathrm{W}$ at $635 \mathrm{~nm}$ for a reverse bias of $3.8 \mathrm{~V}$. The photodetector shows a data rate of $300 \mathrm{Mb} / \mathrm{s}$ for $860 \mathrm{~nm}$ and up to $510 \mathrm{Mb} / \mathrm{s}$ for $635 \mathrm{~nm}$. The responsivity for $635 \mathrm{~nm}$ light is larger than for $860 \mathrm{~nm}$, which is not the case for classical PDs (section 4.2 ) where $850 \mathrm{~nm}$ has a higher responsivity than $650 \mathrm{~nm}$. The shorter wavelength has a smaller penetration depth; so less diffusing carriers are generated, and a smaller part is canceled by subtraction at shorter wavelengths.

In [25] a $75 \mu \mathrm{mx} 75 \mu \mathrm{m}$ SML N+/NW/P-sub photodetector was integrated in $180 \mathrm{~nm}$ CMOS technology with TIA and an equalizer. The PD had a net responsivity of $0.052 \mathrm{~A} / \mathrm{W}$ at $2.6 \mathrm{~V}$ bias and a $-3 \mathrm{~dB}$ bandwidth of $700 \mathrm{MHz}$. A metal layer over the circuits was used to block incident light. A higher metal layer would have resulted in a lower parasitic capacitance, but the incident light is blocked more effectively by using a metal close to the active circuitry.

SML photodetector was integrated with TIA, equalizer, LA, and output buffer also in $130 \mathrm{~nm}$ CMOS process [84]. The SML PD had $0.05 \mathrm{~A} / \mathrm{W}$ responsivity at $1.5 \mathrm{~V}$ supply voltage. Simulations showed that the TIA with the integrated SML PD achieves the $-3 \mathrm{~dB}$ bandwidth of $1.2 \mathrm{GHz}$ at 850 $\mathrm{nm}$ light. An adaptive equalizer was integrated to increase the optical receiver bandwidth. The fully integrated optical receiver achieved a measured bandwidth of $5.9 \mathrm{GHz}$ at $850 \mathrm{~nm}$ by implementing an equalizer. An optical sensitivity of $-3.2 \mathrm{dBm}$ for a BER of $10^{-12}$ was reported. Medici simulations were performed in [2] for a $80 \mu \mathrm{mx} 80 \mu \mathrm{m}$ differential PD (same as SML PD) in $90 \mathrm{~nm}$ CMOS technology. The DC responsivity varied between $0.052 \mathrm{~A} / \mathrm{W}$ for 18 fingers and $0.034 \mathrm{~A} / \mathrm{W}$ for 34 fingers. More fingers correspond to more interconnection overhead, and a slightly lower responsivity. The intrinsic $-3 \mathrm{~dB}$ bandwidth ranged from $1 \mathrm{GHz}$ for 18 fingers to 4.4 $\mathrm{GHz}$ for 34 fingers. More fingers per diode mean less distance for the diffusing carriers resulting in a faster response.

\subsection{Triple Junction Photodetector}

To cancel out the slow diffusion components, N+/PW photodiode can be created inside a DNW as shown in Fig. 19. The electrons photogenerated in the P-sub diffusion region are collected by the DNW and shorted to the supply contact of DNW.

The holes photogenerated inside the DNW diffusion region are collected by the PW and shorted to the ground contact of the PW [13]. A part of the electrons generated in the PW diffusion region is collected by the DNW and shorted to the supply contact of DNW. A part of the electrons generated in the PW diffusion region is collected by the N+/PW photodiode [13]. Since the distance traveled by these electrons by diffusion before being collected by the $\mathrm{N}+$ cathode is short (PW depth $\leq 1 \mu \mathrm{m})$ a high bandwidth $(\geq 2 \mathrm{GHz})$ is expected, where $\mathrm{Dn}=10 \mathrm{~cm}^{2} / \mathrm{s}$ inside the $\mathrm{PW}$. 
The structure of the interrupted finger can be mixed with the triple junction PD to obtain a triple well interrupted N-Finger photodiode [13]. The N+/PW/DNW/P-sub photodiode with interrupted $\mathrm{N}+$ finger anode with a total size of $65 \mu \mathrm{m} \times 65 \mu \mathrm{m}$ was implemented in 130nm CMOS technology. Using Medici, the photodiode achieves a simulated bandwidth of $9.7 \mathrm{GHz}$ while having a responsivity of $2.3 \mathrm{~mA} / \mathrm{W}$ for $850 \mathrm{~nm}$. Due to the efficient cancellation of the diffusive carriers in this type of the photodiode; the bandwidth is increased on the expense of a very low responsivity. A triple junction structure (see Fig. 19) was exploited in an APD. The APD was designed and fabricated with $65 \mathrm{~nm}$ standard CMOS technology without any process modification. The reverse bias voltage was applied to the N+/PW junction, and photocurrents were extracted from the $\mathrm{N}+$ (cathode) contact located inside the PW (anode). STI between $\mathrm{P}+$ and $\mathrm{N}+$ regions prevents premature edge breakdown. An optical window having the area of $30 \mu \mathrm{m}$ by $30 \mu \mathrm{m}$ was formed by blocking the salicide process. It achieved the responsivity of $2.94 \mathrm{~A} / \mathrm{W}$ for $850 \mathrm{~nm}$ light and a $-3 \mathrm{~dB}$ bandwidth of $3.2 \mathrm{GHz}$ at the reverse bias voltage of $10.6 \mathrm{~V}$ [15].

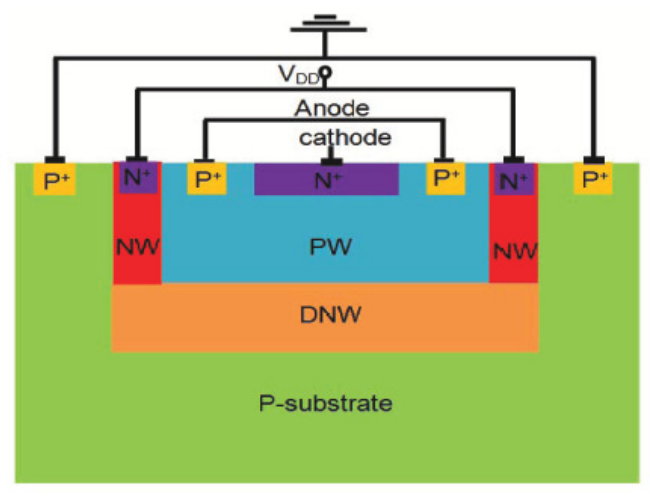

Fig. 19: Cross section of triple junction photodetector.

\subsection{Comparison of Nanometer CMOS Photodiodes}

For comparison, a Figure of Merit (FoM) is defined for the photodiodes using their performance parameters such as junction capacitance per area ( $\mathrm{C}, \mathrm{fF} \mathrm{mm} 2)$, leakage current ( $\mathrm{Ir}, \mathrm{mA})$, bandwidth $(\mathrm{BW}, \mathrm{MHz})$ and responsivity $(\mathrm{R}, \mathrm{A} / \mathrm{W})$ as well as the used technology node (L, nm) [13].

$$
F_{O} M_{1}=\left(\frac{B W(M H z) \cdot R(A / W)}{C\left(\frac{f F}{\mu m^{2}}\right) \cdot L^{2}(n m) \cdot I(\mu A)}\right) \cdot 1000
$$

$\mathrm{FoM}_{1}$ is inversely proportional to the square of the technology feature length; because of the effect of higher doping concentration, which increases the junction capacitance. Also as technology scales down, the number of dielectric layers in the stack on top of the die increase, this increases optical interference and reduces somewhat the quantum efficiency [13]. Table 1 shows a comparison between PD structures for $850 \mathrm{~nm}$ wavelength. $\mathrm{FoM}_{2}$ is calculated without the effect of dark current. $\mathrm{FoM}_{3}$ is calculated without considering the effect of dark current and technology scaling, i. e. without considering L. 
Electrical and Electronics Engineering: An International Journal (ELELIJ) Vol 3, No 2, May 2014

Table 1: Comparison between different photodiodes structures

\begin{tabular}{|c|c|c|c|c|c|c|c|c|}
\hline & $\mathrm{BW}(\mathrm{MHz})$ & $\mathrm{C}(\mathrm{fF}) / \mu \mathrm{m}^{2}$ & $\mathrm{R}(\mathrm{A} / \mathrm{V})$ & $\mathrm{L}(\mathrm{nm})$ & $I_{f}(\mu \mathrm{A})$ & $\overline{\mathrm{F} 0 \mathrm{M}_{1}}$ & $\mathrm{~F} 0 \mathrm{~N}_{2}$ & $\mathrm{~F}_{0} \mathrm{M}_{3}(M)$ \\
\hline $\mathrm{P}+/ \mathrm{NTV} / \mathrm{P}$-sub APD with guard [8] & 2500 & $0.3^{*}$ & 20.3 & 130 & 3 & $3336.3^{6}$ & 10009 & 169.16 \\
\hline N+/PIV/DNT/P-sub APD [15] & 3200 & $0.46^{*}$ & 2.94 & 65 & 0.3 & $16134^{4}$ & 4840.2 & 20.45 \\
\hline $\mathrm{P}^{+} / \mathrm{NWVP}$-sub ADPD [16] & 700 & 0.63 & 0.840 & 40 & 3 & $194.4^{10}$ & 583.2 & 0.933 \\
\hline NIV-PIV/P-sub [21] & 1600 & 0.138 & 0.74 & 180 & 0.1 & $2648^{7}$ & 264.8 & 8.579 \\
\hline NIV/P-sub APD [8] & 100 & $0.138^{*}$ & 4.68 & 130 & 2 & $100.3^{11}$ & 200.6 & 3.391 \\
\hline NIV/P-sub [14] & 40 & 0.048 & 0.34 & 40 & 0.05 & $3541^{5}$ & 177.05 & 0.283 \\
\hline$\overline{\mathrm{N}+/ \mathrm{NIV} / \mathrm{P} \text {-epi/Psub PIN [7] }}$ & 1610 & 0.053 & 0.135 & 180 & 0.00048 & $263690^{\circ}$ & 126.57 & 4.1 \\
\hline $\mathrm{N}+/ \mathrm{P}-$ sub $[14]$ & 40 & 0.039 & 0.16 & 40 & 0.05 & $1923^{8}$ & 102.5 & 0.164 \\
\hline P+/P-epi/DNIV/P-sub PIN [7] & 3130 & 0.163 & 0.141 & 180 & 0.00046 & $180369^{2}$ & 82.97 & 2.7 \\
\hline Lateral P+/P-/N+PIN [7] & 1850 & 0.171 & 0.137 & 180 & 0.00043 & $106385^{3}$ & 45.74 & 1.48 \\
\hline N+/NIV/P-sub SMIL [25] & 700 & 0.088 & 0.052 & 180 & $=$ & - & 12.76 & 0.413 \\
\hline PIV/DNT/P-sub DPD [16] & 9 & 0.387 & 0.33 & 40 & 0.1 & $47.9^{12}$ & 4.79 & 0.0076 \\
\hline
\end{tabular}

\section{Conclusion}

A comparative study for recently published photodiodes structures fabricated in nanometre CMOS technology were presented. By arranging Table 1 in a way ordered with respect to $\mathrm{FoM}_{2}$, the top (best) three PDs are APDs. Also by using $\mathrm{FoM}_{3}$ the avalanche PDs still have the first and second best performance. The APDs have high responsivity and high bandwidth on the expense of high dark current. The high dark current involved with the APD will increase the PD noise and will reduce the optical receiver sensitivity. By considering the dark current $\left(\mathrm{FoM}_{1}\right)$, the best three PDs are PIN-PDs because of high bandwidth and the low dark current.

\section{REFERENCES}

[1] H. Zimmermann, Integrated Silicon Optoelectronics. Springer-Verlag Berlin Heidelberg, second edition, 2010.

[2] C.Hermans and M. Steyaert, Broadband Opto-Electrical Receivers in Standard CMOS, Springer ,Netherlands, 2007.

[3] K. Schneider and H. Zimmermann, Highly Sensitive Optical Receivers. Springer Berlin Heidelberg, 2006.

[4] H. Zimmermann, Silicon Optoelectronic Integrated Circuits. Springer, Berlin Heidelberg, 2004.

[5] S.-H. Huang, W.-Z. Chen, Y.-W. Chang, and Y.-T. Huang, "A 10-Gb/s OEIC with Meshed SpatiallyModulated Photodetector in 0.18um CMOS Technology," IEEE J. Solid-State Circuits, vol. 46, No.5, p. 11581169, 2011.

[6] H. Zimmermann, H. Dietrich, A. Ghazi, P. Seegebrecht, "Fast CMOS-Integrated Finger Photodiodes for aWide Spectral Range," ESSDERC, pp. 435-438, 2002.

[7] B. Ciftcioglu, L. Zhang, J. Zhang, J. R. Marciante, J. Zuegel, R. Sobolewski, and Hui Wu, "Integrated Silicon PIN Photodiodes Using Deep N-Well in a Standard 0.18um CMOS Technology," Journal of Lightwave Technology, vol. 27, no. 15, pp. 3303-3323, 2009.

[8] Myung-Jae Lee and Woo-Young Choi, "Performance Comparison of Two Types of Silicon Avalanche Photodetectors Based on N-well/P-substrate and P+/N-well Junctions Fabricated With Standard CMOS Technology," Journal of the Optical Society of Korea, vol. 15, no. 1, pp. 1-3, 2011.

[9] E. Palik, Handbook of Optical Constants of Solids. Academic, Orlando, 1985.

[10] J. Sturm, Photodiode Modeling and Optoelectronic Integrated Circuits for Optical -Data - Storage Applications. Vienna University of Technology, PhD. Thesis, 2006. 
[11] A. Polzer, K. Schneider-Hornstein, J. Dong, P. Kostov, and H. Zimmermann, "Investigation of Triplejunction Photodetector in 90nm CMOS Technology," PROC. EUROSENSORS XXV, Procedia Engineering, vol. 25, p. 864867, 2011.

[12] F. Tavernier and M. Steyaert, "High-speed optical receivers with integrated photodiode in $130 \mathrm{~nm}$ CMOS," Solid-State Circuits, IEEE Journal of, vol. 44, pp. 2856 -2867, oct. 2009.

[13] B. Nakhkoob, S. Ray, and M. Hella, "High speed photodiodes in standard nanometer scale CMOS technology: a comparative study," Opt. Express, vol. 20, pp. 11256-11270, 2012.

[14] M. Atef, A. Polzer, and H. Zimmermann, "High-Speed Photodiodes in 40nm Standard CMOS Technology," Sensors \& Actuators: A. Physical, 2013.

[15] M.-J. Lee and W.-Y. Choi, "A silicon avalanche photodetector fabricated with standard CMOS technology with over 1THz gain-bandwidth product," Opt. Express, vol. 18, no. 23, p. 2418924194, 2010.

[16] M. Atef, A. Polzer, and H. Zimmermann, "Avalanche Double Photodiode in 40nm sandard CMOS Technology,” IEEE Journal of Quantum Electronics, vol.49, no.3, pp.350-356, 2013.

[17] Alexis Rochas, Alexandre R. Pauchard, Pierre-A. Besse, Dragan Pantic, Zoran Prijic, and Rade S. Popovic, "Low-Noise Silicon Avalanche Photodiodes Fabricated in Conventional CMOS Technologies,” IEEE TRANSACTIONS ON ELECTRON DEVICES, vol. vol. 49, no. 3, pp. 387-394, 2002.

[18] T. K. Woodward and A. V. Krishnamoorthy, "1 Gbit/s CMOS Photoreceiver with Integrated Photodetector Operating at 850 nm," IEE Electronics Letters, vol. 34, no.12, p. 12521253, 1998.

[19] T.K. Woodward, A.V. Krishnamoorthy, "1Gbit/s CMOS Photoreceiver with Integrated Detector Operating at 850nm,” Electron. Lett., vol. 34, no.12, pp. 1252-1253, 1998.

[20] T. K. Woodward and A. V. Krishnamoorthy, "1 Gbit/s Integrated Optical Detectors and Receivers in Commercial CMOS Technologies,” IEEE J. Sel. Top. Quantum Electron, vol. 5, no.2, p. 146156, 1999.

[21] Wei-Kuo Huang, Yu-Chang Liu, and Yue-Ming Hsin, "A High-Speed and High-Responsivity Photodiode in Standard CMOS Technology," IEEE Photonics Technology Letters, vol. 19, no. 4, pp. 197-199, 2007.

[22] D. Coppee, W. Pan, R. Vounckx, and M. Kuijk, “The Spatially Modulated Light Detector," Optical Fiber Conf., OSA Tech. Dig. Ser., p. 315316, 1998.

[23] J. Genoe, D. Coppee, J. H. Stiens, R. A. Vounckx, andM. Kuijk, "Calculation of The Current Response of The Spatially Modulated Light CMOS Detector," IEEE Trans. Electron. Dev., vol. 48, no.9, p. 18921902, 2001.

[24] Maarten Kuijk, Daniel Coppee, and Roger Vounckx, "Spatially Modulated Light Detector in CMOS with Sense-Amplifier Receiver Operating at $180 \mathrm{Mb} / \mathrm{s}$ for Optical Data Link Applications and Parallel Optical Interconnects Between Chips," IEEE Journal of Selected Topics in Quantum Electronics, vol. 4, no.6, pp. 1040-1045, 1998.

[25] T.-C. Kao, F. Musa, and A. Carusone, "A 5-gbit/s cmos optical receiver with integrated spatially modulated light detector and equalization," Circuits and Systems I: Regular Papers, IEEE Transactions on, vol. 57, pp. $2844-2857$, nov. 2010. 